\title{
GROUND-LEVEL DETECTION OF AURORAL KILOMETRIC RADIATION
}

\author{
J. LaBelle*, and R. R. Anderson ${ }^{\dagger}$
}

\begin{abstract}
The Earth's aurorae radiate away up to $1 \%$ of their energy in the form of radio waves, called Auroral Kilometric Radiation (AKR). The mechanism responsible for the emission, the electron cyclotron maser (ECM), produces similar emissions at other planets, in the solar atmosphere, and in astrophysical systems. AKR was not unambiguously identified until the 1970's because its detection requires a suitably instrumented satellite. The ECM theory predicts radiation beamed outward that cannot penetrate the increasing magnetic field and electron density near the Earth. Nevertheless, there have been observations over the years of AKR-like radio signals detected by ground-based, rocket-borne, and low-earth orbiting satellite-borne instruments, raising the question of whether a mechanism exists by which AKR can penetrate to low altitudes. Here we show the first unambiguous evidence that AKR indeed penetrates to low altitudes on occasions. We identified three examples of AKR-like emissions detected with a ground-based radio receiver at South Pole Station, Antarctica, during a 9-day interval in July, 2004, when the Geotail satellite, monitoring AKR, had a field of view including the auroral field lines above the station. The AKR-like emissions detected at ground-level have the same frequenc-time structure as simultaneous AKR emissions detected on Geotail 115,000-190,000 km away from the Earth. Slight differences in the frequency extent of the emissions at the two locations can be explained by, for example, plasmaspheric screening of the emissions detected by Geotail. These observations represent the first coincident detections of AKR in space and on the ground. They require the existence of an as-yet unidentified mechanism to produce the ground-level emissions which are not predicted by ECM theory, they suggest that previous AKR-like emissions observed at low altitudes may indeed be AKR, and they require revision of the widely-held view that AKR is only detectable from space.
\end{abstract}

${ }^{*}$ Department of Physics and Astronomy, Dartmouth College, Hanover, New Hampshire, USA

$\dagger$ Department of Physics and Astronomy, University of Iowa, Iowa City, IA 52242, USA 
